\title{
Non-targeted Parallel Cascade Selection Molecular Dynamics Using Time-localised Prediction of Conformational Transitions in Protein Dynamics
}

\author{
Ryuhei Harada ${ }^{*}, \dagger$, Vladimir Sladek ${ }^{*} ₫$, and Yasuteru Shigeta* ${ }^{*} \dagger$ \\ ${ }^{\dagger}$ Center for Computational Sciences, University of Tsukuba 1-1-1 Tennodai, Tsukuba, \\ Ibaraki 305-8577, Japan \\ $\S$ Institute of Chemistry - Centre for Glycomics, Dubravska cesta 9, 84538 Bratislava, \\ Slovakia \\ ¥Agency for Medical Research and Development (AMED), Chiyoda-ku, Japan \\ Corresponding authors: Ryuhei Harada, Vladimir Sladek, and Yasuteru Shigeta \\ E-mail: ryuhei@ccs.tsukuba.ac.jp,sladek.vladimir@savba.sk, \\ shigeta@ccs.tsukuba.ac.jp
}




\section{Figure S1}
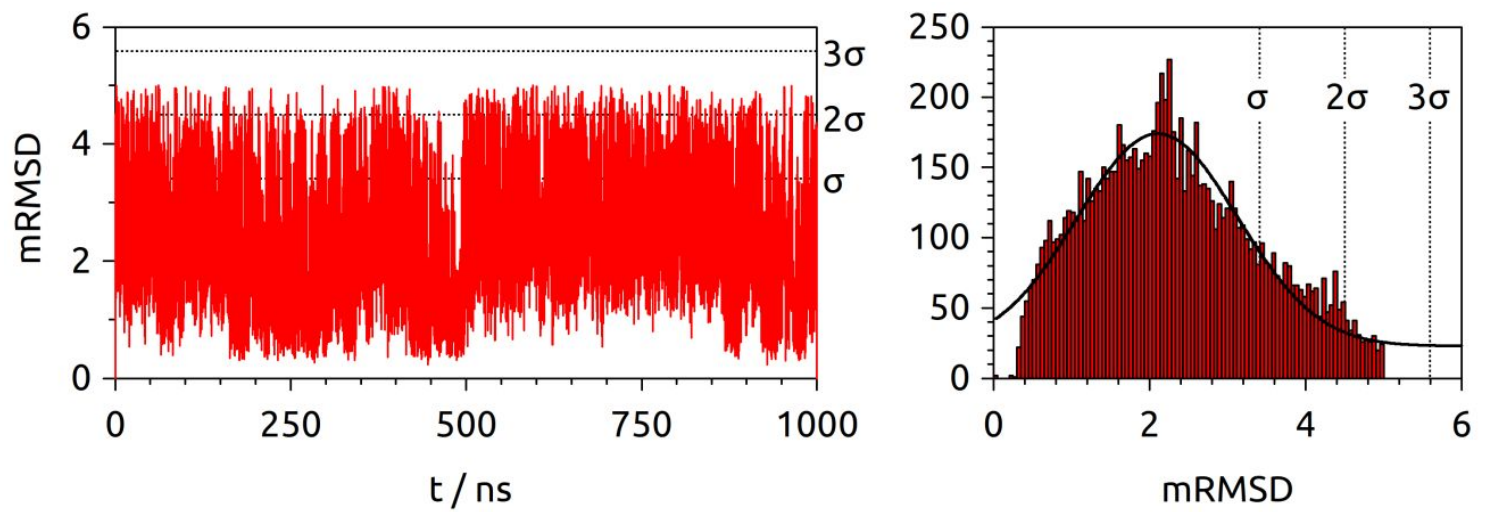

mRMSD time series for deca-alanine during $1 \mu \mathrm{s}$ MD simulation of folding (time step for the recorded data points is $100 \mathrm{ps}$ ). The right-hand-side plot depicts the histogram of mRMSD. The size of the reference domain was $l^{-}=1$. Multiples of standard deviation $\sigma$ are shown in both plots. 
Figure S2


mRMSD histograms for selected nt-PaCS-MD cycles of chignolin folding simulation. Each cycle is 100 ps long with 1 ps time step for the recording of data points. The size of the reference domain was $l^{-}=1$. Multiples of standard deviation $\sigma$ are shown. 


\section{Figure S3}

a)

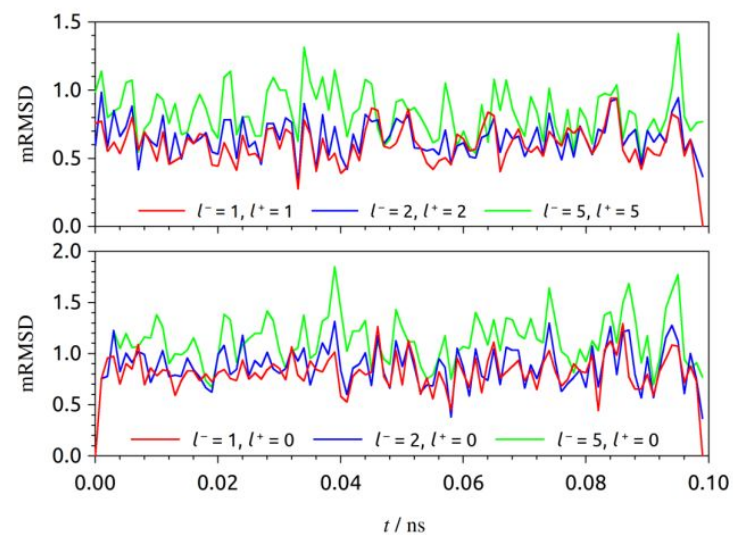

b)

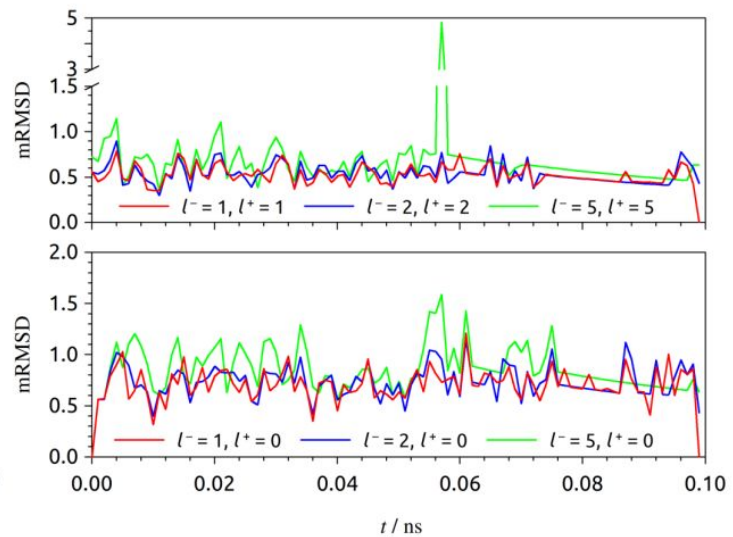

mRMSD time series for two selected nt-PaCS-MD cycles of chignolin folding simulation; column a) the zeroth cycle and column b) the $325^{\text {th }}$ cycle. The effect of varying reference domain size is shown. 


\section{Figure S4}

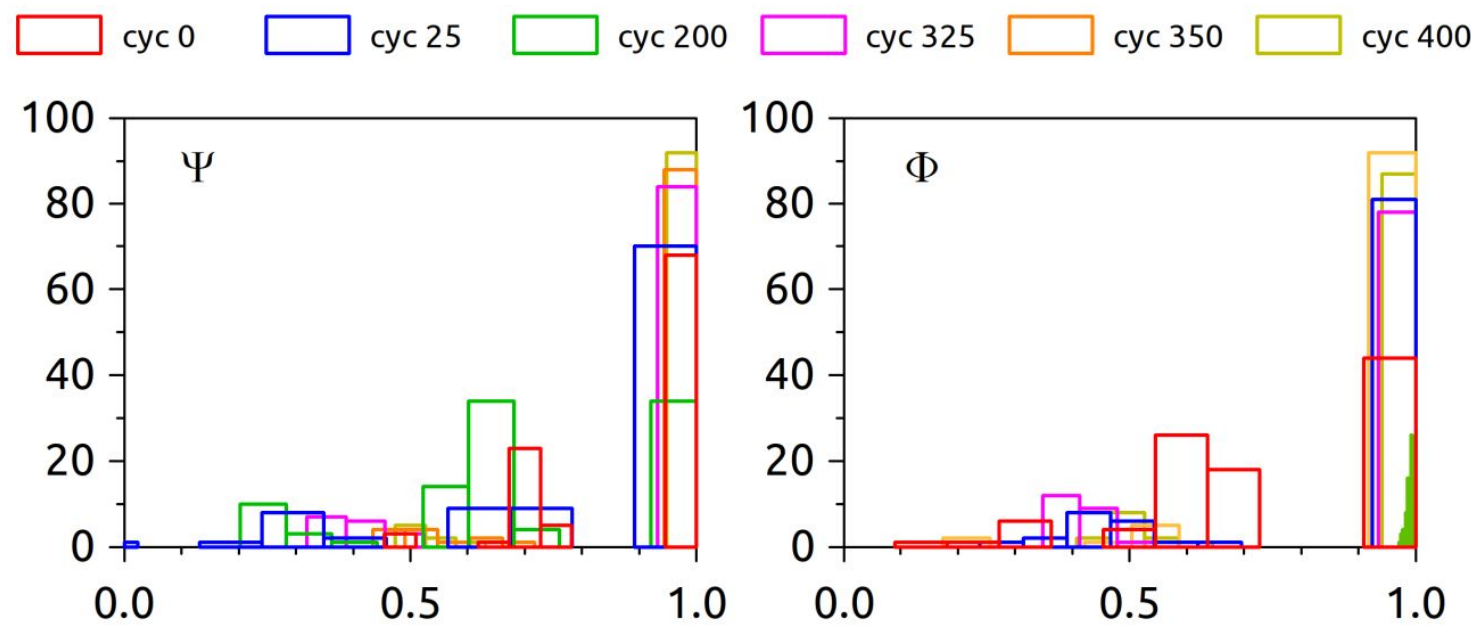

$\Phi, \Psi$ histograms for selected nt-PaCS-MD cycles of chignolin folding simulation. The size of the reference domain was $l^{-}=1$. 


\section{Figure S5}

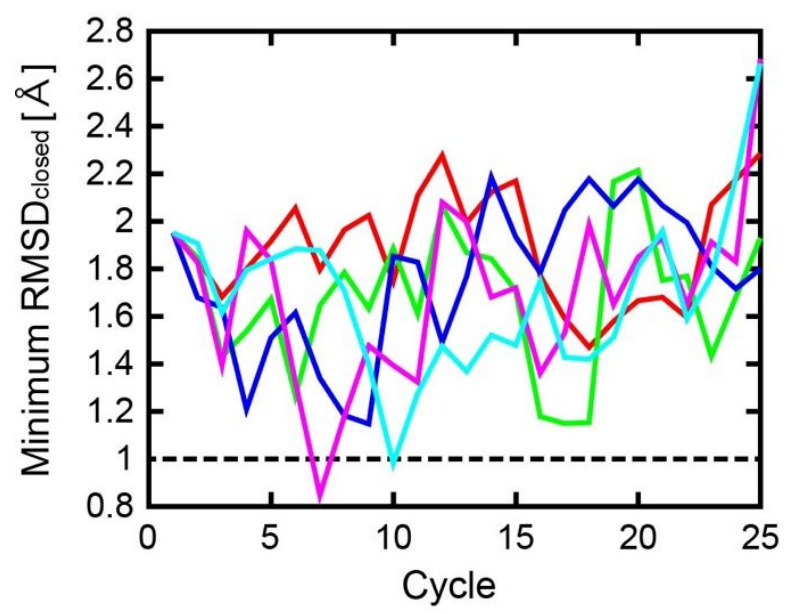

Profiles of minimum root-mean square deviation measured from the closed structure of T4 lysozyme $\left(\mathrm{RMSD}_{\text {closed }}\right)$ for the 25 cycles. Each color represents each nt-PaCS-MD (independent five runs) using the long-time interval (50 ps) for saving snapshots in the short-time MD simulations. The dashed line is a threshold for identify the closed structure. 


\section{Figure S6}

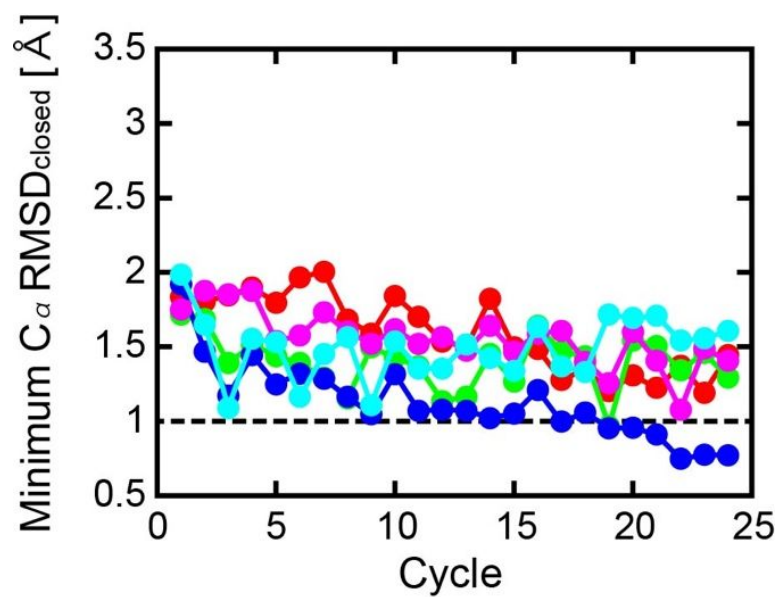

A set of profiles of minimum $C_{\alpha} \mathrm{RMSD}_{\text {closed }}$ of five nt-PaCS-MD runs based on $\Phi$ and $\Psi\left(N_{\text {initial }}=20\right)$ for the 25 cycles. Each color represents one nt-PaCS-MD run. The dashed line represents a threshold of $C_{\alpha} \mathrm{RMSD}_{\text {closed }}$ for identifying the closed structure of T4 lysozyme. 
Figure S7

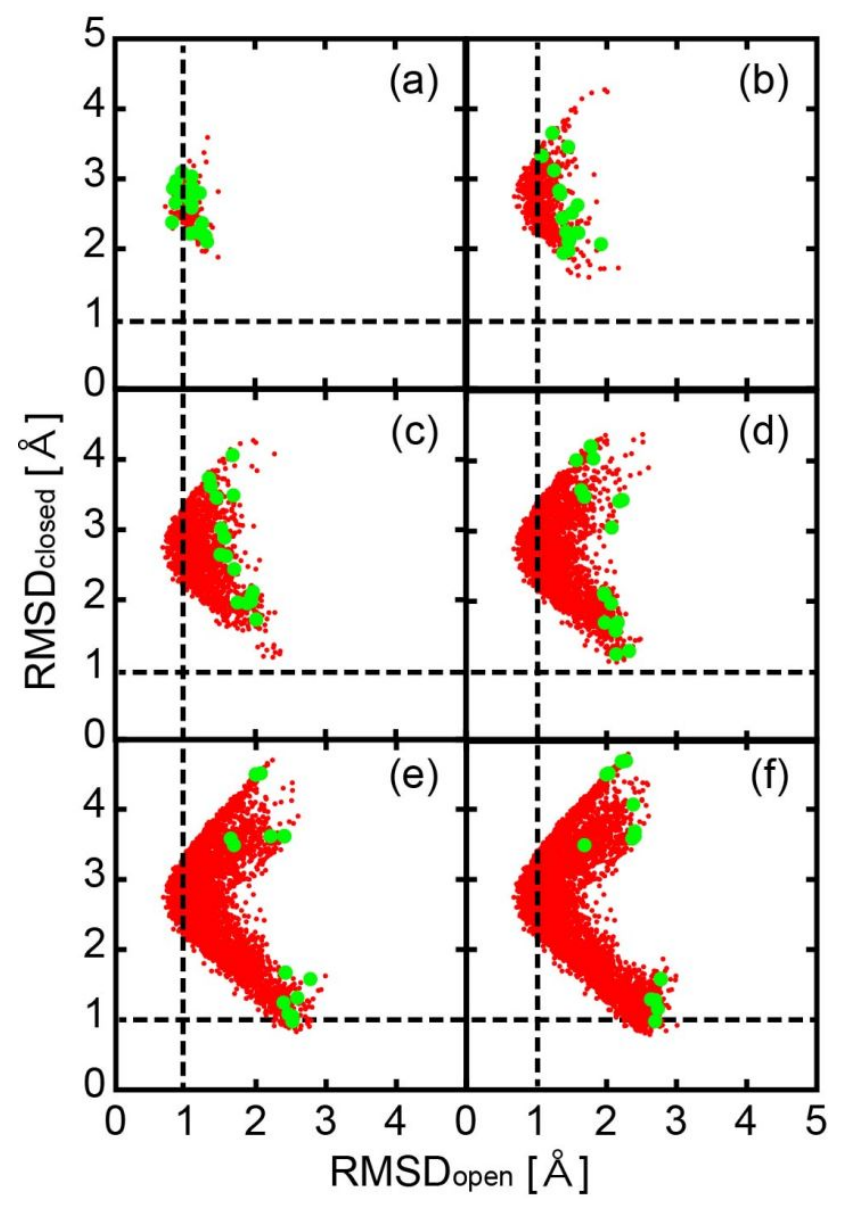

(red) Projections of accumulated protein configurations generated by one of the ntPaCS-MD runs based on mRMSD $\left(N_{\text {initial }}=20\right)$ as a function of $\mathrm{C}_{\alpha} \mathrm{RMSD}_{\text {open }}$ and $\mathrm{C}_{\alpha}$ RMSD ${ }_{\text {closed }}$ after (a) the $2^{\text {nd }}$, (b) the $5^{\text {th }}$, (c) the $10^{\text {th }}$, (d) the $15^{\text {th }},(\mathrm{e})$ the $20^{\text {th }}$, and (f) the $25^{\text {th }}$ cycles, respectively. (green) Projections of 20 Initial structures selected at each cycle. The dashed lines show thresholds for identifying the open and closed structures of T4 lysozyme. 


\section{Figure S8}
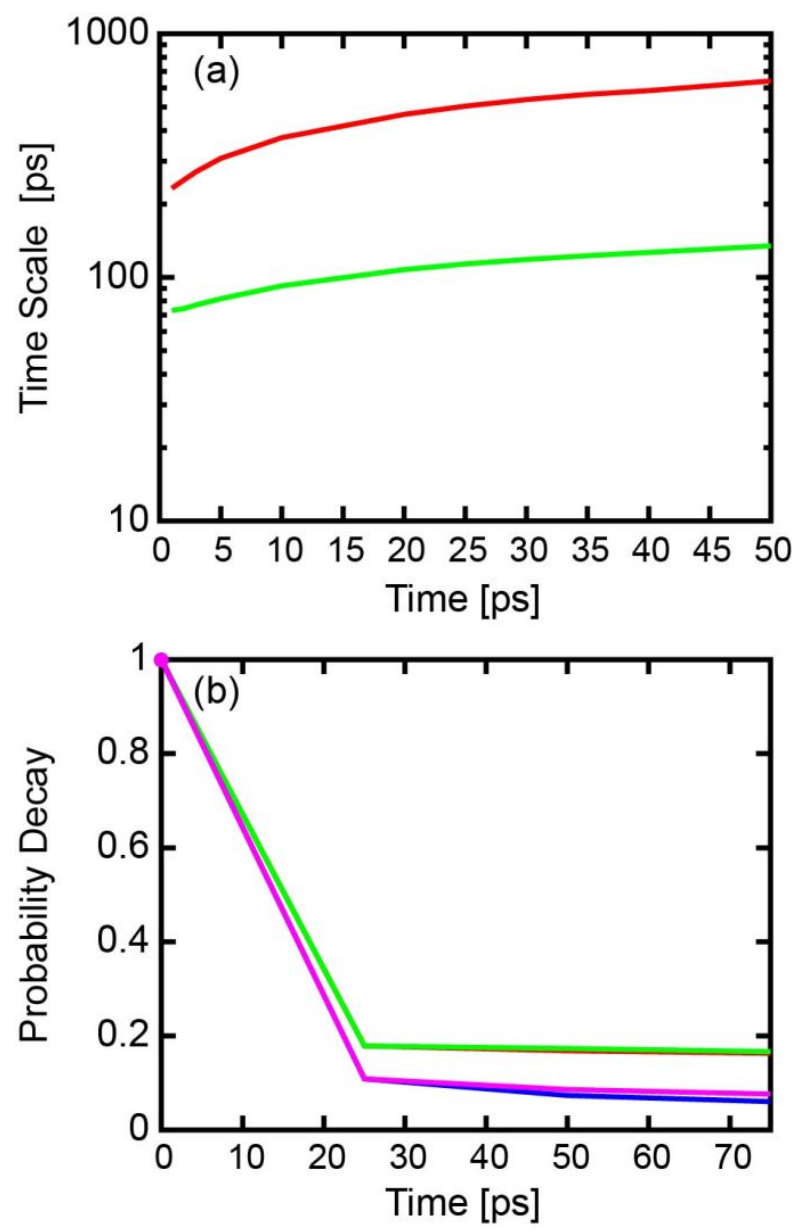

(a) Implied relaxation time scales of MSM using the $k$-means algorithm with 50 cluster centers as a function of lag time for the $1^{\text {st }}$ and $2^{\text {nd }}$ eigenvalues. (b) ChapmanKolmogorov test of the MSM conducted at $\tau=25$ ps with 50 microstates defined by the $k$-means clustering for (two) major metastable states. (green/magenta) The probability decay of the major metastable states predicted by the MSM construction and (red/blue) those estimated from the trajectories of the five nt-PaCS-MD trials based on mRMSD $\left(N_{\text {initial }}=20\right)$. 
Figure S9

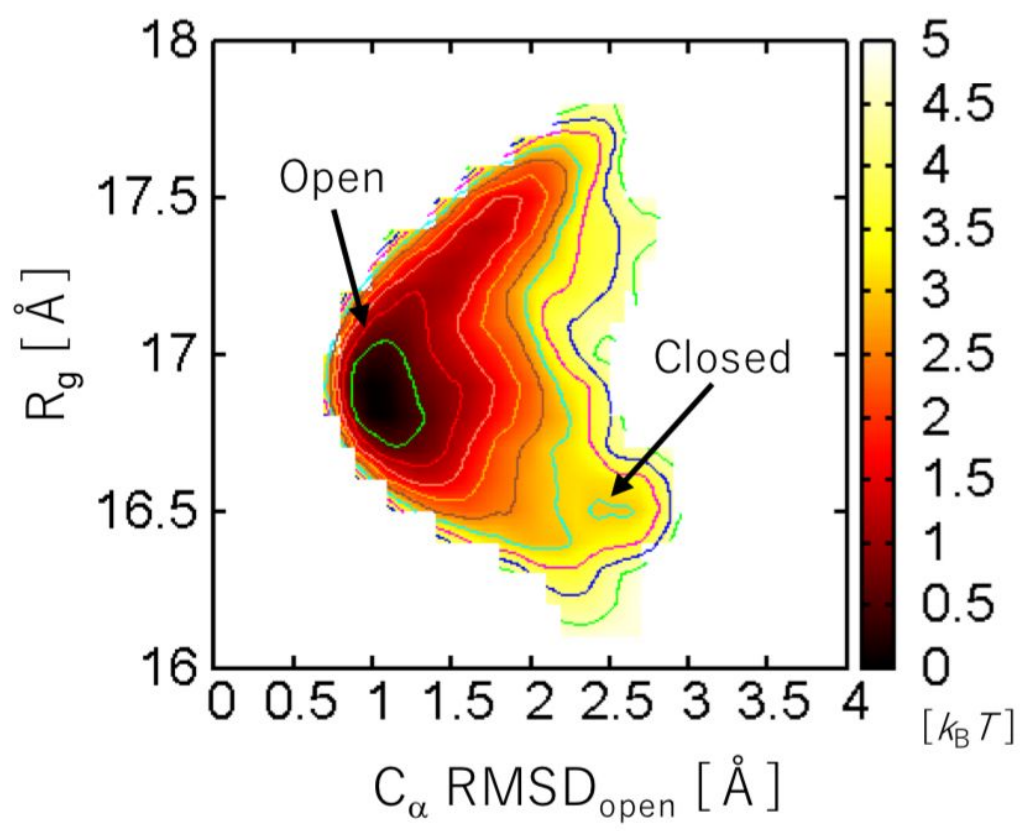

Free energy landscape (FEL) calculated by the Markov State Model (MSM) as a function of RMSD measured from the open structure of T4 lysozyme $\left(\mathrm{RMSD}_{\text {open }}\right)$ and radius of gyration $\left(R_{\mathrm{g}}\right)$. 